\title{
Development of Fast and Soft Landing System for Quadcopter Drone using Fuzzy Logic Technology
}

\author{
Ashish Kumar ${ }^{1}$, Sugjoon Yoon ${ }^{2}$ \\ ${ }^{1}$ Sejong University, Seoul, Republic of Korea, ashishkumar.aero@ gmail.com \\ ${ }^{2}$ Sejong University, Seoul, Republic of Korea, sjyoon@ @ejong.edu
}

\begin{abstract}
The popularity of Drones, also known as unmanned aerial vehicles (UAVs), are highly increasing in various fields because of its multi-skilled performance and operational flexibility. Landing of the drone is the most difficult part of a flight. Literature review reveals, there are many kinds of landing controllers are available for drones but those controllers take more time for landing a drone. In this paper, we developed a fast as well as a soft landing control system for quadcopter drone using fuzzy logic technology. More specifically, we designed a fuzzy logic based controller in MATLAB/Simulink and implemented it to Raspberry pi. The Raspberry pi is connected with an ultrasonic sensor for altitude calculation. Here we used Pixhawk as the main flight controller, and the Pixhawk receives the real-time landing control commands from the Raspberry pi. Several landing tests were conducted using the designed fuzzy landing control system, and the flight test data shows that the quadcopter landed softly with minimum time than the other existing landing control technologies.
\end{abstract}

Key words: Fuzzy logic controller, Pixhawk, Quadcopter landing, Raspberry pi.

\section{INTRODUCTION}

Nowadays, a lot of researches going on for drone exploration [1]-[18]. There are different types of drones are available on the market, mainly fixed-wing [6], single-rotor [7] and multi-rotor [8]. The multi-rotor drones are the most widely used type due to its applicability. There are also many types of multi-rotor drones, including quadcopter [9], hexacopter [10], octocopter [11]. According to mechanical simplicity and performance, the quadcopter is the most demanded drone [1]-[3]. The flight controller is the main part of any drones, including quadcopter. Currently, the Pixhawk controller has been hugely used as a flight controller for drones due to its cheap cost and best performance [12]. Pixhawk is an open-source autopilot hardware and its supports PX4 and ArduPilot flight stacks.
Different types of landing control systems are available for quadcopter because the landing of the quadcopter is a tricky task. Usually, PID control techniques are mostly used for the automatic quadcopter landing. Literature review shows that the existing landing control systems take more amount of time for the quadcopter landing [13]-[17]. Recently, the quadcopter is widely used for military purposes, like enemy attack, surveillance, transportation, search and rescue, etc. These kinds of military applications need a very fast response, so the fast, as well as the safe landing, is required for military applications. And, if the landing time is less, we can also save the battery charges. Muhammad Talha et al. [18] proposed a time-efficient auto-landing controller for quadcopter using a fuzzy logic-based technique. Their landing controller takes about $20 \mathrm{~s}$ for landing from 500 to $0 \mathrm{~cm}$ altitude.

This paper proposed a fast and soft landing control system for quadcopter using fuzzy logic technology [18], [19]. The fuzzy controller is designed using MATLAB/Simulink. Altitude and landing velocity are inputs and throttle command is the output of the fuzzy controller. Herein we implemented the designed fuzzy controller in Raspberry pi and connected with the Pixhawk controller. An ultrasonic sensor is attached to the Raspberry pi for altitude measuring. In this work, Pixhawk is the main flight controller, so the raspberry pi sends the real-time landing control commands to the Pixhawk for the throttle adjustment. Our designed fuzzy landing controller is verified through several quadcopter landing tests and also we tested the quadcopter landing using PD control technology and Pixhawk automatic land option. Here we compared the flight test data of our proposed fuzzy landing controller with the flight test data of the PD controller and Pixhawk automatic land. The comparison results show that the designed fuzzy landing controller takes very less amount of time for the safe landing than the other landing control techniques.

\section{QUADCOPTER DYNAMICS}

The quadcopter motion has been described by translational motion $(x, y$, and $z)$ and rotational motion $(\phi, \theta$, and $\psi)$. The geometry of the quadcopter is shown in Figure 1. 


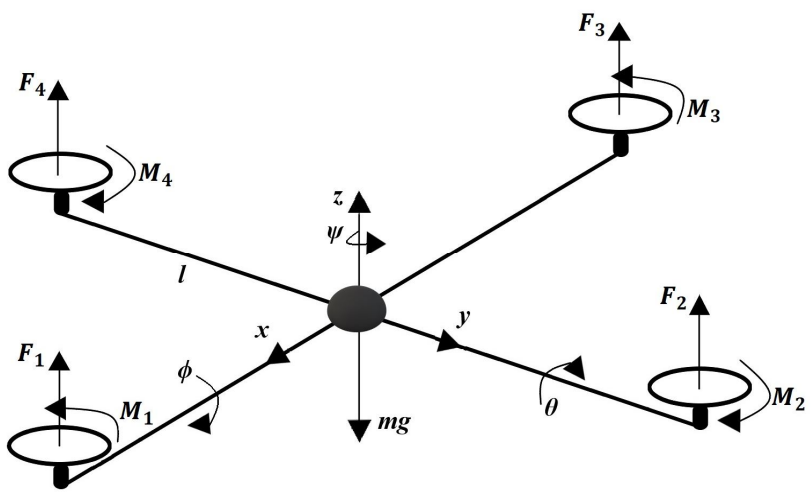

Figure 1: Geometry of quadcopter

The equation of motion of the quadcopter can be expressed as [20] [21]:

$$
\begin{aligned}
& \ddot{x}=\left(\frac{U_{1}}{m}\right)(\cos (\phi) \sin (\theta) \cos (\psi)+\sin (\phi) \sin (\psi)) \\
& \ddot{y}=\left(\frac{U_{1}}{m}\right)(\cos (\phi) \sin (\theta) \sin (\psi)-\sin (\phi) \cos (\psi)) \\
& z=\left(\frac{U_{1}}{m}\right)(\cos (\phi) \cos (\theta))-\theta \\
& \ddot{\phi}=\left(\left(I_{y y}-I_{z z}\right) \theta \dot{\psi}+\left(U_{z}-I_{r} \theta \omega_{y}\right)\right) \frac{1}{I_{x x}} \\
& \dot{\theta}=\left(\left(I_{z z}-I_{x x}\right) \dot{\phi} \dot{\psi}+\left(U_{2}-I_{r} \dot{\phi} \omega_{r}\right)\right) \frac{1}{I_{y y}} \\
& \dot{\psi}=\left(\left(I_{x x}-I_{y y}\right) 6 \phi+\left(U_{4}\right) \frac{1}{I_{z z}}\right.
\end{aligned}
$$

where $g$ is the gravity value, $\mathrm{m}$ is the total mass of quadcopter, $\omega_{r}=\omega_{1}-\omega_{2}+\omega_{3}-\omega_{4}, I_{x x}, I_{y y}$ and $I_{z z}$ are the moments of inertia and $I_{r}$ is the inertia of the motor.

\section{FUZZY LOGIC CONTROLLER DESIGN FOR QUADCOPTER LANDING}

The fuzzy logic control contains five main processes which are, input defining, fuzzification, fuzzy rule base, defuzzification, and output defining. Considering the time consuming, the fuzzy logic controller is better than the PID controller for automatic landing [18]. In this section, we described the design procedure of the fuzzy controller for the fast and soft landing of the quadcopter. Herein the fuzzy controller has two inputs and one output. Altitude and landing velocity are inputs and throttle command is the output of the fuzzy controller (see Figure 2).

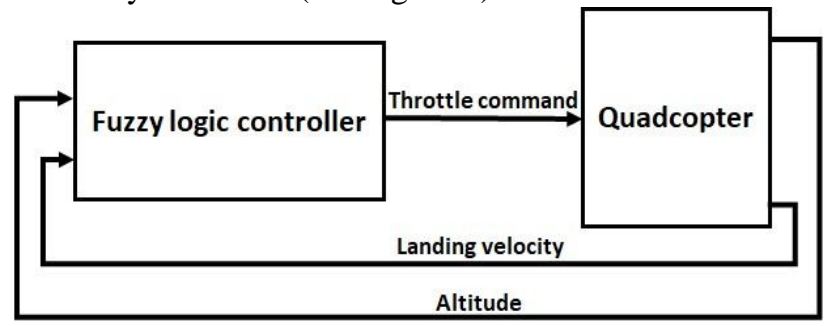

Figure 2: Outline of the fuzzy landing controller.
Table 1: Four stages of the fuzzy landing controller.

\begin{tabular}{|c|c|}
\hline \multirow{2}{*}{ Stage - 5} & Altitude of quadcopter \\
\cline { 2 - 2 } & 1.6 to $1.2 \mathrm{~m}$ \\
\hline Stage -4 & 1.6 to $0.8 \mathrm{~m}$ \\
\hline Stage -3 & 1.2 to $0.4 \mathrm{~m}$ \\
\hline Stage -2 & 0.8 to $0.0 \mathrm{~m}$ \\
\hline Stage -1 & 0.4 to $0.0 \mathrm{~m}$ \\
\hline
\end{tabular}

Table 2: Fuzzy control rule base for the fast and soft landing.

\begin{tabular}{|c|c|c|c|c|c|}
\hline \multirow{2}{*}{ Altitude } & \multicolumn{4}{|c|}{ Landing Velocity } \\
\cline { 2 - 6 } & DVB & DVS & NV & UVS & UVB \\
\hline Stage - 5 & UVB & UVS & DVB & DVB & DVB \\
\hline Stage - 4 & UVS & UVS & DVB & DVB & DVB \\
\hline Stage - 3 & UVS & UVS & DVS & DVS & DVB \\
\hline Stage - 2 & UVB & UVS & NV & DVS & DVS \\
\hline Stage - 1 & UVB & UVB & NV & DVS & DVS \\
\hline
\end{tabular}

In this paper, the fuzzy controller has five stages and it starts from $1.6 \mathrm{~m}$ to $0 \mathrm{~m}$ altitude (see Table 1). The fuzzy control rule base for the fast and soft landing is shown in Table 2 . DVB and DVS represent downward velocity big and downward velocity small, respectively. UVB and UVS denoted upward velocity big and upward velocity small, respectively and $\mathrm{NV}$ implies the normal velocity for the soft landing.

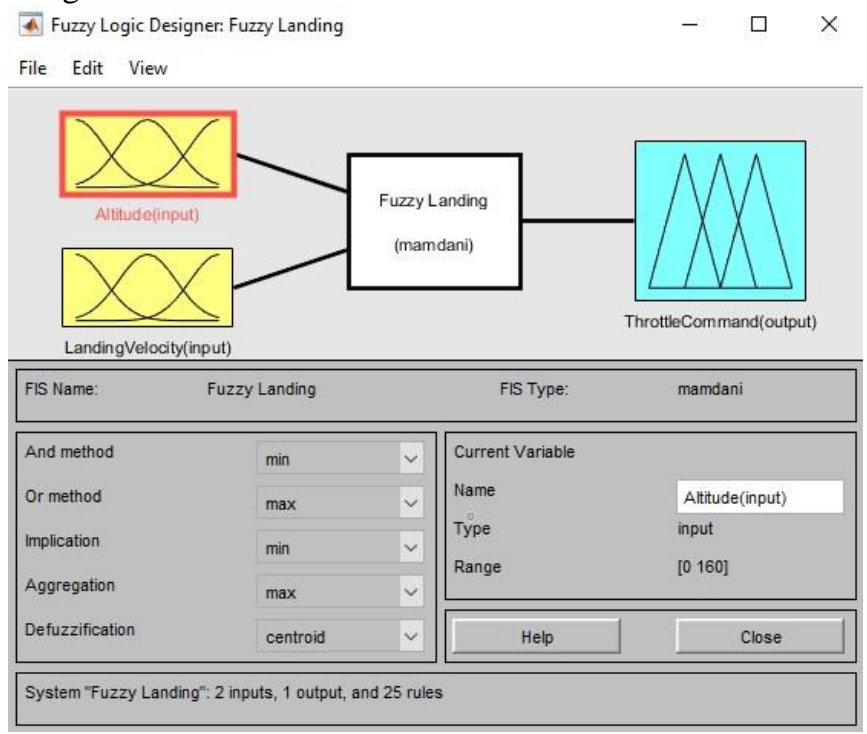

Figure 3: Fuzzy logic designer in MATLAB/Simulink. 


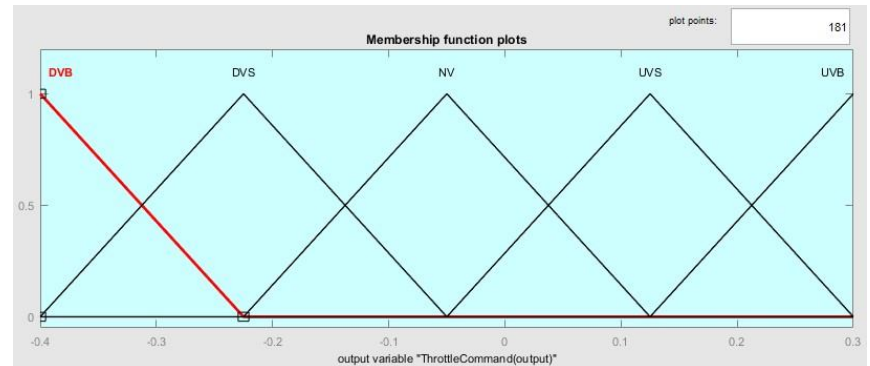

Figure 4: Output membership function (throttle command) of the fuzzy controller.

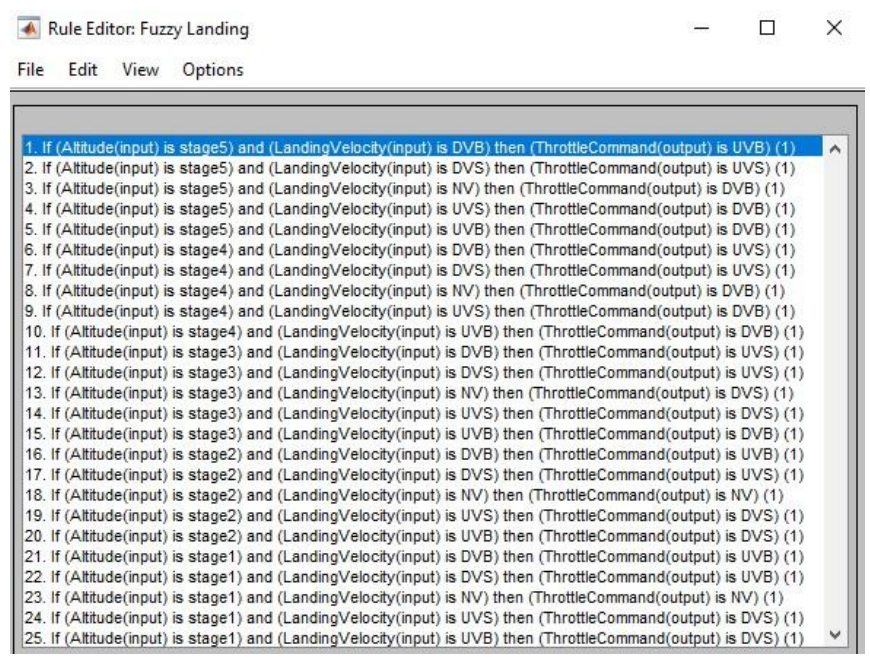

Figure 5: Fuzzy control rules for the quadcopter landing.

This paper used MATLAB/Simulink for designing the fuzzy controller. We designed the input and output membership function, and the rule base using Fuzzy toolbox in the MATLAB/Simulink (see figure 3). Triangular membership functions are used here. The output membership function (throttle command) of the fuzzy controller is shown in Figure 4. Herein the fuzzy controller is designed with 25 rules for the quadcopter landing. Figure 5 illustrates the 25 fuzzy control rules. The fully designed fuzzy control system is shown in figure 6.

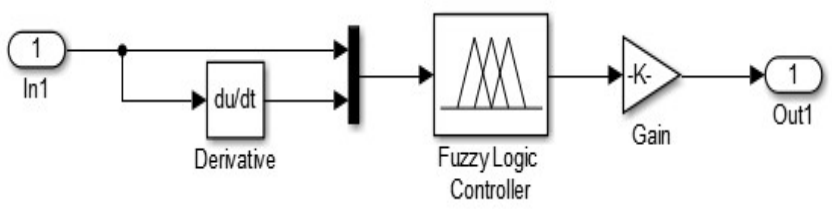

Figure 6: Designed fuzzy landing controller in MATLAB/Simulink.

\section{PD CONTROLLER DESIGN FOR QUADCOPTER LANDING}

The proportional and derivative (PD) control is a conventional control technique. Here we designed a quadcopter landing controller using PD technology. The general equation for PD can be expressed as;

$$
U(t)=K_{p} e(t)+K_{d} \frac{d e(t)}{d t}
$$

where $K_{p}$ is the proportional gain, $K_{d}$ is the derivative gain and ' $e$ ' implies the error. The designed PD landing control system is shown in Figure 7.

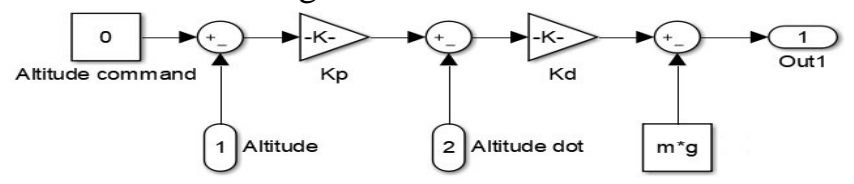

Figure 7: Designed PD landing controller in MATLAB/Simulink

\section{SIMULATION SETUP AND RESULTS}

For simulation, we developed a quadcopter MATLAB/Simulink model with the parameters of the F450 Spider quadcopter. The parameters of the F450 Spider quadcopter are mentioned in Table 3. The developed quadcopter model contains an attitude controller using PD technology, and it consists of three controllers which are, roll, pitch and yaw controller. The designed simulation model of the quadcopter (F450 Spider) is shown in figure 8. Here we implemented the designed fuzzy logic landing controller and PD landing controller to the quadcopter simulation model separately and conducted several quadcopter landing simulation tests.

Table 3: Parameters of F450 Spider Quadcopter.

\begin{tabular}{|c|c|}
\hline Quadcopter parameters & Values \\
\hline Gross weight + battery & $2 \mathrm{~kg}$ \\
\hline Propellers dimension \& pitch & 10 inch $\times 3.8$ \\
\hline No of blade & 2 \\
\hline Frame diagonal length & $450 \mathrm{~mm}$ \\
\hline $\begin{array}{l}\text { Moments of Inertia }\left(\mathrm{I}_{\mathrm{xx}}, \mathrm{I}_{\mathrm{yy}},\right. \\
\left.\mathrm{I}_{\mathrm{zz}}\right)\end{array}$ & $0.0035,0.0035,0.005 \mathrm{~kg} . \mathrm{m}^{2}$ \\
\hline Thrust coefficient & $9.8 \times 10^{-6} \mathrm{~N} / \mathrm{m}^{2}$ \\
\hline Drag coefficient & $1.6 \times 10^{-7}$ \\
\hline
\end{tabular}

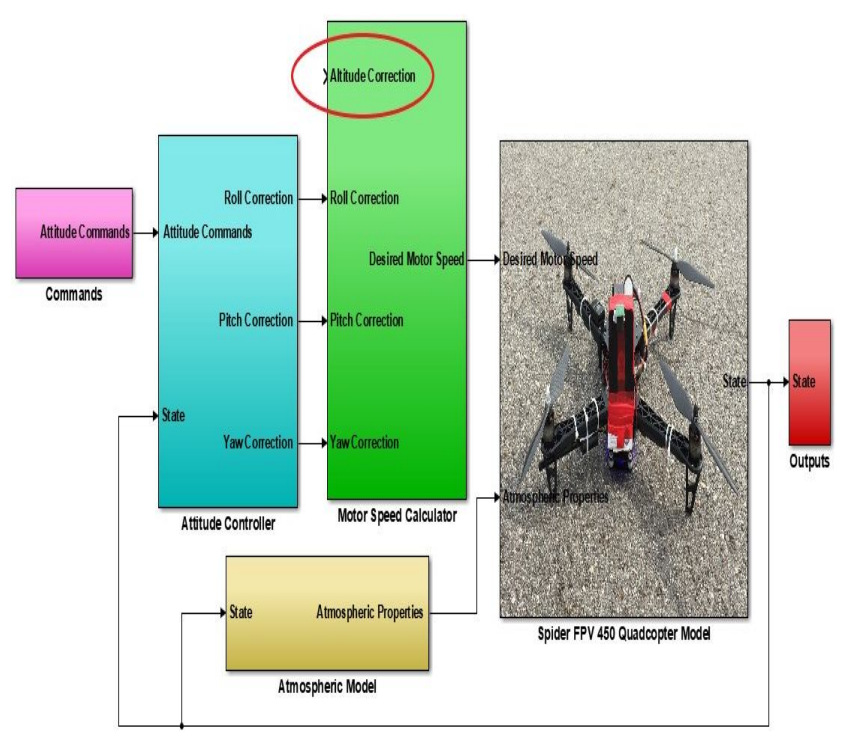

Figure 8: Simulation model of F450 Spider Quadcopter in MATLAB/Simulink. 
The following Figures 9 and 10 illustrate that the landing simulation test results of the quadcopter using the designed fuzzy and PD controller. The landing position of the quadcopter shown in Figure 9, and Figure 10 show that the landing velocity of the quadcopter. From the results we can see, the PD controller takes more than 10 seconds than the fuzzy controller for the quadcopter safe landing (from 4 to 0 $\mathrm{m})$. Therefore in the simulation results, the fuzzy controller is more time-saving than the PD controller.

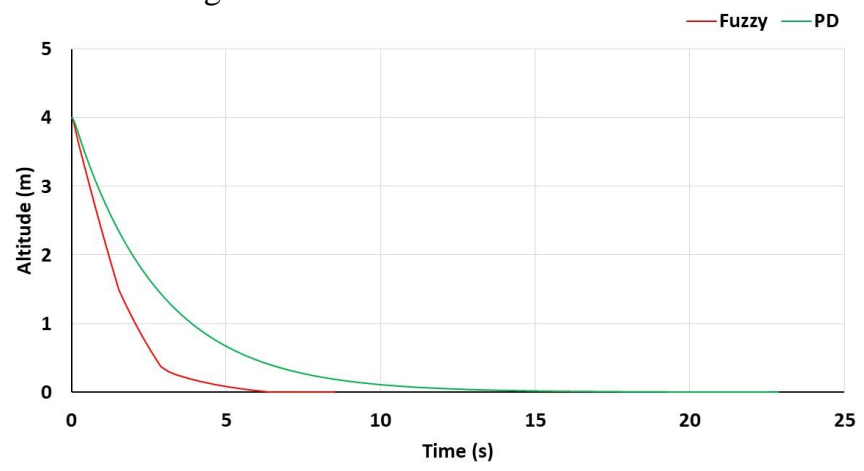

Figure 9: Simulation results of quadcopter landing position using the fuzzy and PD controller.

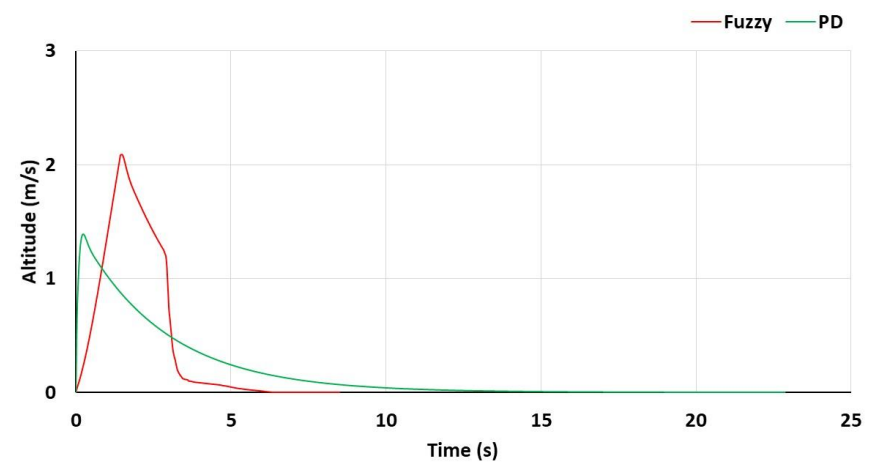

Figure 10: Simulation results of quadcopter landing velocity using the fuzzy and PD controller.

\section{EXPERIMENTAL SETUP AND RESULTS}

In this section, we presented the real experimental landing test of the quadcopter using the designed fuzzy controller and the PD controller. To do so, we implemented the designed controllers in the Raspberry pi. Here we used a Raspberry pi 3 with a PmodMAXSONAR sensor. The PmodMAXSONAR is an ultrasonic range finder sensor, here the sensor is used for measuring the altitude of the quadcopter. In this work, we used F450 Spider Quadcopter. The principal flight controller of the quadcopter is Pixhawk. So we connected the Raspberry pi to Pixhawk by serial connection. During the quadcopter landing, the Raspberry pi sends the throttle commands to Pixhawk for the landing. The Spider F450 Spider Quadcopter with the landing experimental setup is shown in Figure 11.

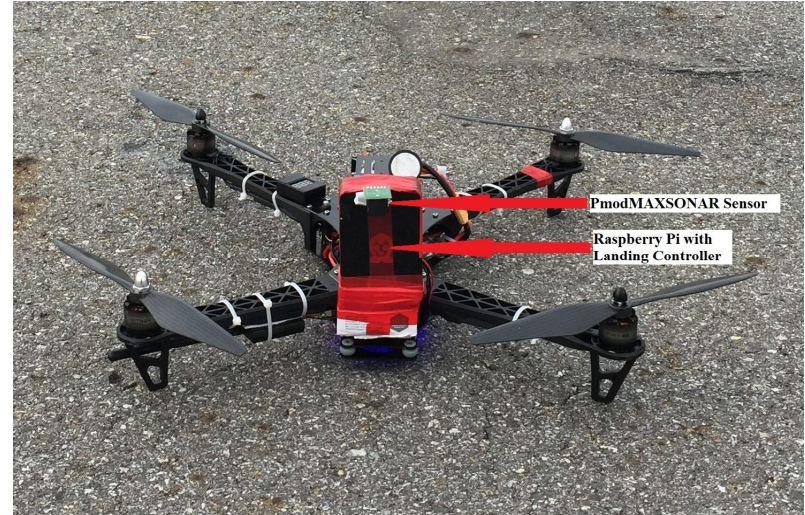

Figure 11: F450 Spider Quadcopter with landing experimental setup.

In this paper, we conducted three cases of quadcopter landing tests, which are, landing by, the fuzzy controller, the PD controller, and the Pixhawk automatic land option. For the Pixhawk automatic land, we connected a GPS module and installed the Arducopter program to the quadcopter. The Arducopter is an open-source autopilot platform written in $\mathrm{C}++$. Herein, the landing by the fuzzy and PD controller is worked without the GPS and the Pixhawk automatic land is worked with the GPS. The experimental results of the quadcopter landing by, the fuzzy controller, the PD controller, and the Pixhawk automatic land are shown in Figure 12. From the result we can see, the quadcopter landed softly in three cases and the fuzzy controller takes only 10.5 seconds for the safe landing from 4 to $0 \mathrm{~m}$ altitude and, the PD controller and Pixhawk automatic land takes 19.5 and 16 seconds, respectively. There are more than 5 seconds of time differences between the fuzzy and the other two landing cases. Figures 13 and 14 illustrate that the roll and pitch angle of the quadcopter during the landing in the case of the fuzzy controller. The picture of the quadcopter landing test (fuzzy controller) is shown in Figure 15. The experimental result shows that the designed fuzzy logic controller is more time-efficient than the PD controller and Pixhawk automatic land.

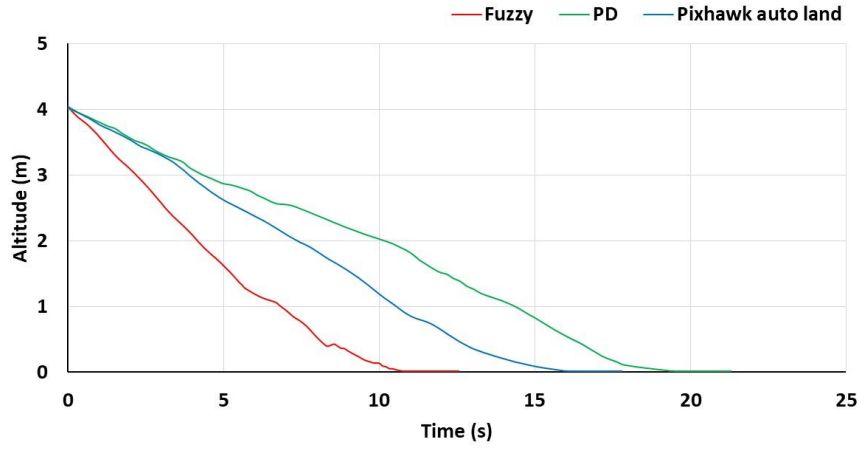

Figure 12: Experimental results of quadcopter landing by, the fuzzy controller, the PD controller, and the Pixhawk automatic land. 


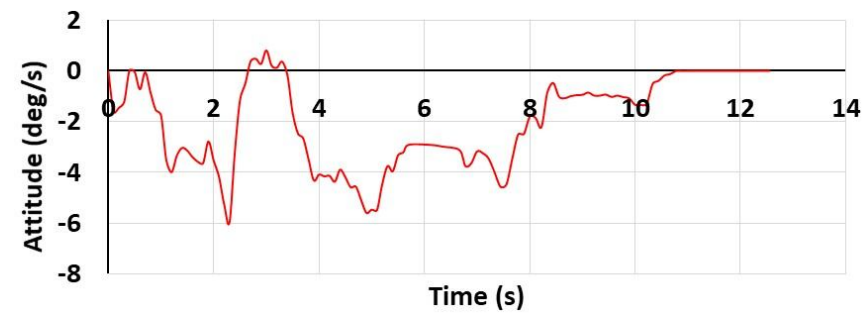

Figure 13: Roll angle of quadcopter during the landing (fuzzy controller).

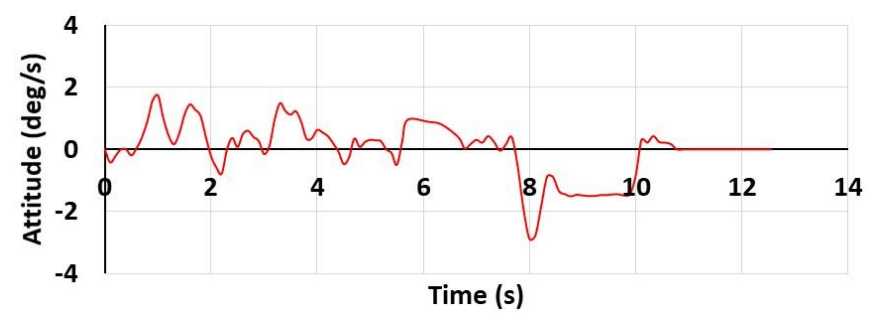

Figure 14: Pitch angle of quadcopter during the landing (fuzzy controller).
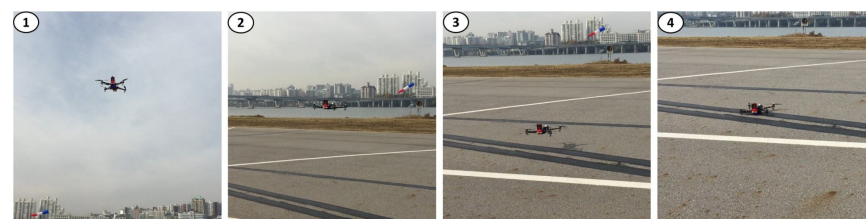

Figure 15: F450 Spider Quadcopter landing test (fuzzy controller).

\section{CONCLUSION}

This paper proposed a fuzzy logic control technology for the fast and soft landing of a quadcopter. Herein we designed a fuzzy controller in MATLAB/Simulink and implemented it to Raspberry pi. In the designed fuzzy controller, altitude and velocity are inputs and throttle command is the output. By using an ultrasonic sensor with Raspberry pi, we calculated the real-time altitude of the quadcopter. The Raspberry pi is directly connected to the principal flight controller Pixhawk by serial connection. For landing, the Raspberry pi sends the real-time throttle commands to the Pixhawk. We conducted several quadcopter landing flight tests with our designed fuzzy control system. The experimental results show that the fast and soft landing achieved through the proposed fuzzy landing system. For comparison, here we also carried out the quadcopter landing test using the PD control technology and Pixhawk automatic land option. From the all flight test results, and the literature review studies, we proposed that the designed fuzzy logic based controller can land the quadcopter securely with the less amount of time than the other existing landing control technologies.

\section{ACKNOWLEDGEMENT}

This work is supported by the Korea Agency for Infrastructure Technology Advancement (KAIA) grant funded by the
Ministry of Land, Infrastructure, and Transport (Grant 18CTAP-C142966-01).

\section{REFERENCES}

1. Ashish Kumar, Christophe Mondon, and Sugjoon Yoon. Development of Mixed Reality Simulation based on an Unmanned Aerial Vehicle, CHIRA 2019 Proceedings of the 3rd International Conference on Computer-Human Interaction Research and Applications, Vienna, Austria, September 2019, pp. 89-96.

2. Ashish Kumar, Sugjoon Yoon, Amrith M, Thianesh U K, V.R.S.Kumar. Mixed reality simulation of a quadcopter with a DHEM device for planet landers for soft landing-A review, International Journal of Advanced Science and Technology; Vol. 28, No. 14, pp. 157-171, 2019.

3. Ashish Kumar. Mixed Reality Simulation Based on an Unmanned Aerial Vehicle and X-plane Flight Simulator, Republic of Korea, Patent Application No. 1615010612, December 2019 (Submitted).

4. De Guzman J.E.C, Delos Santos I.M, Pulido D.A.A, Renes D.R.T, Roque M.A.C. Semi Autonomous Quadcopter for Early Rat Infestation Detection. International Journal of Advanced Trends in Computer Science and Engineering. Vol. 8, No. 2, pp. 241-245, 2019.

https://doi.org/10.30534/ijatcse/2019/22822019

5. Serhii Semenov, Denys Voloshyn and Ahmed Najat Ahmed. Mathematical Model of the Implementation Process of Flight Task of Unmanned Aerial Vehicle in the Conditions of External Impact. International Journal of Advanced Trends in Computer Science and Engineering. Vol. 8, No. 1, pp. 7-13, 2019.

6. Randal Beard, Derek Kingston, Morgan Quigley, Deryl Snyder, Reed Christiansen, Walt Johnson, Timothy McLain and Michael A. Goodrich. Autonomous Vehicle Technologies for Small Fixed-Wing UAVs, Journal of Aerospace Computing Information and Communication, Vol. 2, No. 1, pp. 92-108, 2005. DOI: 10.2514/1.8371

7. O. C. Carholt, E. Fresk, G. Andrikopoulos and G. Nikolakopoulos. Design, Modelling and Control of a Single Rotor UAV, 24th Mediterranean Conference on Control and Automation (MED), Athens, Greece, June 2016, pp. 840-845. DOI: 10.1109/MED.2016.7536015.

8. Robert Niemiec and Farhan Gandhi. Control and Performance of a Reconfigurable Multicopter. Journal of Aircraft, Vol. 55, No. 5, pp. 1855-1866, 2018. https://doi.org/10.2514/1.C034731

9. Karam Eliker, Guoqing Zhang, Said Grouni, and Weidong Zhang, An Optimization Problem for Quadcopter Reference Flight Trajectory Generation, Journal of Advanced Transportation, Vol. 2018, pages. 15, 2018. https://doi.org/10.1155/2018/6574183

10. Radek Baránek, and František Šolc, Modelling and control of a hexa-copter, Proceedings of the 13th 
International Carpathian Control Conference (ICCC), High Tatras, Slovakia, May 2012. DOI: 10.1109/CarpathianCC.2012.6228609

11. Karisma Trinanda Putra, Rama Okta Wiyagi and Muhamad Yusvin Mustar, Precision Landing System on H-Octocopter Drone Using Complementary Filter, International Conference on Audio, Language and Image Processing (ICALIP), Shanghai, China, 2018. pp. 283-287. DOI: 10.1109/ICALIP.2018.8455553

12. Lorenz Meier, Petri Tanskanen, Friedrich Fraundorfer, and Marc Pollefeys. PIXHAWK: a system for autonomous flight using onboard computer vision. Proceedings of IEEE International Conference on Robotics and Automation, Shanghai, China, May 2011; pp. 2992-2997.

13. Yingcai $\mathrm{Bi}$ and Haibin Duan. Implementation of Autonomous Visual Tracking and Landing for a Low-Cost Quadrotor, Optik - International Journal for Light and Electron Optic, Vol. 124, pp. 3296-3300, 2013. https://doi.org/10.1016/j.ijleo.2012.10.060

14. Roman Barták, Andrej Hrasko and David Obdrzalek. A controller for autonomous landing of AR.Drone, 26th Chinese Control and Decision Conference, Changsha, China, May 2014. DOI: 10.1109/CCDC.2014.6852167

15. Patrick Benavidez, Josue Lambert, Aldo Jaimes and Mo Jamshidi. Landing of an Ardrone 2.0 quadcopter on a mobile base using fuzzy logic, World Automation Congress (WAC), Waikoloa, HI, USA, August 2014. DOI: 10.1109/WAC.2014.6936156

16. Yi Feng, Cong Zhang, Stanley Baek, Samir Rawashdeh and Alireza Mohammadi. Autonomous Landing of a UAV on a Moving Platform Using Model Predictive Control, Drones (MDPI journal), 2, 34, 2018. https://doi.org/10.3390/drones2040034

17. Nguyen Xuan-Mung and Sung-Kyung Hong. Improved Altitude Control Algorithm for Quadcopter Unmanned Aerial Vehicles. Applied Science, 9, 2122, 2019. https://doi.org/10.3390/app9102122

18. Muhammad Talha, Furqan Asghar, Ali Rohan, Mohammed Rabah and Sung Ho Kim. Fuzzy Logic-Based Robust and Autonomous Safe Landing for UAV Quadcopter, Arabian Journal for Science and Engineering, 44, pp. 2627-2639, 2019. https://doi.org/10.1007/s13369-018-3330-z

19. Gennady G. Kalach and Gennady P. Kalach. Navigation System Based on the Fuzzy Logic Expert System, International Journal of Advanced Trends in Computer Science and Engineering. Vol. 8, No.6, pp. 2693-2698, 2019. https://doi.org/10.30534/ijatcse/2019/02862019

20. Alexander Lanzon, Alessandro Freddi and Sauro Longhi. Flight Control of a Quadrotor Vehicle Subsequent to a Rotor Failure, Journal of Guidance, Control, and Dynamics, Vol. 37, No. 2, pp. 580-591, 2014. https://doi.org/10.2514/1.59869

21. Khoa Dang Nguyen and Cheolkeun Ha, Development of hardware-in-the-loop simulation based on Gazebo and Pixhawk for unmanned aerial vehicles, International Journal of Aeronautical and Space Sciences, Vol. 19, No. 1, pp. 238-249, 2018. https://doi.org/10.1007/s42405-018-0012-8 